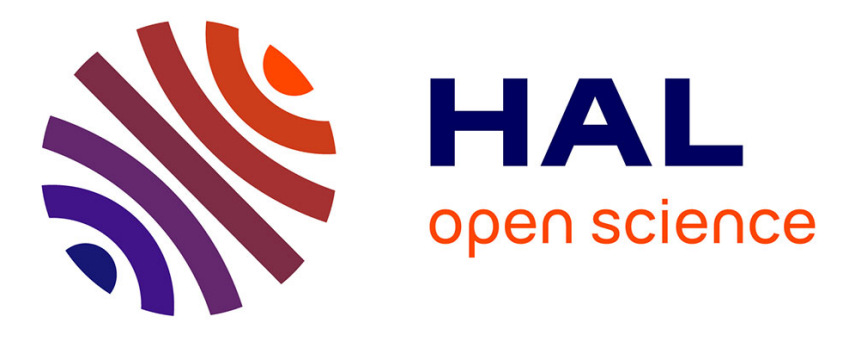

\title{
Harmonics Identification with Artificial Neural Networks: Application to Active Power Filtering
}

Ngac Ky Nguyen, Patrice Wira, Damien Flieller, Djaffar Ould Abdeslam, Jean Merckle

\section{- To cite this version:}

Ngac Ky Nguyen, Patrice Wira, Damien Flieller, Djaffar Ould Abdeslam, Jean Merckle. Harmonics Identification with Artificial Neural Networks: Application to Active Power Filtering. International Journal of Emerging Electric Power Systems, 2011, 12 (5), pp.1-27. 10.2202/1553-779X.2783 . hal00851870

\author{
HAL Id: hal-00851870 \\ https://hal.science/hal-00851870
}

Submitted on 19 Aug 2013

HAL is a multi-disciplinary open access archive for the deposit and dissemination of scientific research documents, whether they are published or not. The documents may come from teaching and research institutions in France or abroad, or from public or private research centers.
L'archive ouverte pluridisciplinaire HAL, est destinée au dépôt et à la diffusion de documents scientifiques de niveau recherche, publiés ou non, émanant des établissements d'enseignement et de recherche français ou étrangers, des laboratoires publics ou privés. 


\section{International Journal of Emerging Electric Power Systems}

Volume 12, Issue 5 2011

Article 1

\section{Harmonics Identification with Artificial Neural Networks: Application to Active Power Filtering}

Ngac Ky Nguyen, Université de Haute Alsace

Patrice Wira, Université de Haute Alsace

Damien Flieller, INSA de Strasbourg

Djaffar Ould Abdeslam, Université de Haute Alsace

Jean Merckle, Université de Haute Alsace

\section{Recommended Citation:}

Nguyen, Ngac Ky; Wira, Patrice; Flieller, Damien; Ould Abdeslam, Djaffar; and Merckle, Jean (2011) "Harmonics Identification with Artificial Neural Networks: Application to Active Power Filtering," International Journal of Emerging Electric Power Systems: Vol. 12: Iss. 5, Article 1. DOI: $10.2202 / 1553-779 X .2783$

Available at: http://www.bepress.com/ijeeps/vol12/iss5/art1

C2011 Berkeley Electronic Press. All rights reserved. 


\title{
Harmonics Identification with Artificial Neural Networks: Application to Active Power Filtering
}

\author{
Ngac Ky Nguyen, Patrice Wira, Damien Flieller, Djaffar Ould Abdeslam, and \\ Jean Merckle
}

\begin{abstract}
This study proposes several high precision selective harmonics compensation schemes for an active power filter. Harmonic currents are identified and on-line tracked by novel Adaline-based architectures which work in different reference-frames resulting from specific currents or powers decompositions. Adalines are linear and adaptive neural networks which present an appropriate structure to fit and learn a weighted sum of terms. Sinusoidal signals with a frequency multiple of the fundamental frequency are synthesized and used as inputs. Therefore, the amplitude of each harmonic term can be extracted separately from the Adaline weights adjusted with a recursive LMS (Least Mean Squares) algorithm. A first method is based on the modified instantaneous powers, a second method optimizes the active currents, and a third method relies on estimated fundamental currents synchronized with the direct voltage components. By tracking the fluctuating harmonic terms, the Adalines learning process allows the compensation schemes to be well suited for on-line adaptive compensation. Digital implementations of the identification schemes are performed and their effectiveness is verified by experiments.
\end{abstract}

KEYWORDS: harmonic identification, harmonic compensation, active power filter, adaline, artificial neural networks, power quality, adaptive control, nonlinear system 
Nguyen et al.: Harmonics Identification with ANNs : Application to APF

\section{Introduction}

In recent years, power quality has become a significant and critical issue. Today's power distribution systems are large and complex. Furthermore, the power production becomes more and more distributed while consumption constantly increases. Nonlinear elements like thyristor-controlled devices and high power converters proliferate in the whole power distribution system and generate harmonic currents. Other types of pollution like unbalance between the phases, reactive power and a bad power factor do not only increase the losses but also produce unwanted disturbances, damages and stress in power distribution systems (Peng, 2001). As a result, electrical pollution is of a raising presence.

Power quality improvement has motivated the development of harmonics compensation schemes such as shunt Active Power Filters (APFs) (Akagi, 1996, Akagi, Watanabe, and Aredes, 2007). These devices estimate the harmonic terms and re-inject them phase-opposite in the power distribution system. For optimal performances, APFs should be able to track the changing harmonics and should be able to adapt their parameters to take into account the time-varying behavior of the power system. Under these conditions, neural-based adaptive controllers are advisable.

An Artificial Neural Network (ANN) provides a computationally efficient way to approximate the nonlinear relationship between multidimensional spaces (Picton, 2000). Basically, an ANN estimates a relationship without any mathematical model and only with representative data. Data are measured inputs and outputs in the case of a real system. Furthermore, by iteratively adjusting their parameters, ANNs are able to take into account complex systems with time-varying behaviors. They have therefore been applied successfully for the identification and control of dynamical complex systems (Norgaard, Ravn, Poulsen, and Hansen, 2000).

The use of adaptive linear neurons called Adalines (Widrow and Walach, 1996) for estimating the harmonic components has been initiated with the work of (Dash, Swain, Liew, and Rahman, 1996). Adalines are able to directly estimate the Fourier coefficients of nonlinear currents corrupted by a random noise. A unified ANN architecture has thus been proposed in (Ould Abdeslam, Wira, Mercklé, Flieller, and Chapuis, 2007) for the control of a shunt APF. Several neural estimation schemes based on Adalines are employed to on-line learn the load currents expressed in different reference frames. The amplitude of the harmonic terms are deduced and their compensation is achieved. Various approaches have been proposed in the literature and reflect the increasing interest of applying adaptive and neural-based techniques for estimating harmonic components (Azevedo, Ferreira, Martins, and Carvalho, 2008, Nguyen and Liao, 2009, Mohanty, Kumar, Routray, and Kabisatpathy, 2010). Multi-Layer Perceptrons (MLPs) and Radial Basis Func- 
tion Neural Networks (RBFNNs) are investigated in (Mazumdar and Harley, 2008), Probabilistic Neural Networks (PNNs) or Support Vector Machines (SVMs) are combined to other techniques like wavelet-based feature vectors (Dash, Nayak, Senapati, and Lee, 2007) or the S-transform (Panigrahi, Dash, and Reddy, 2009). They are efficient solutions to severe estimation problems event with fluctuating parameters (Zadeh, 2010). However, the learning of complex neural architectures remains stiff when handling real-time systems.

This article proposes three simple neural network schemes for identifying harmonic currents. The proposed schemes use Adalines and are based on specific expressions of powers or currents representative of the distorted currents. In the first scheme, the expression of the modified instantaneous powers are learned by Adalines. In the second scheme, optimal active currents are deduced from instantaneous powers and are estimated by Adalines. In the third scheme, the direct fundamental current is expressed in an appropriate current reference frame and is estimated by an Adaline. Once training is achieved, the Adalines allow to access to the amplitudes of the harmonic terms which can be used for compensation and monitoring. Performances of the neural schemes are discussed and verified through compensating current generation in a shunt APF. Results show that the proposed neural schemes can efficiently identify and remove the harmonic terms. This is confirmed with a dSPACE-based prototype used in experiments.

The paper is organized as follows. Section 2 introduces the instantaneous p-q powers and describes how to calculate them with Adalines for estimating harmonic terms within an APF compensation scheme. New Adaline-based methods for estimating the current harmonics are presented in Section 3. Section 4 verifies the expected features and performances of the proposed neural harmonic identification schemes by means of selected experimental results. In addition, a performance comparison with the original p-q method for estimating the harmonics is provided. Section 5 presents the concluding remarks of this paper.

\section{Harmonics identification and powers interpretation}

\subsection{Instantaneous harmonic tracking}

Many papers have dealt with the definition, identification, characterization, detection, measurement, and compensation of non-sinusoidal and non-periodic currents and powers (Peng, 2001). The Instantaneous Power Theory (IPT) of (Akagi, 1996), also known as the p-q theory, has demonstrated its ability for efficiently describing and modeling power systems. Initially proposed for balanced systems, the 
Nguyen et al.: Harmonics Identification with ANNs : Application to APF

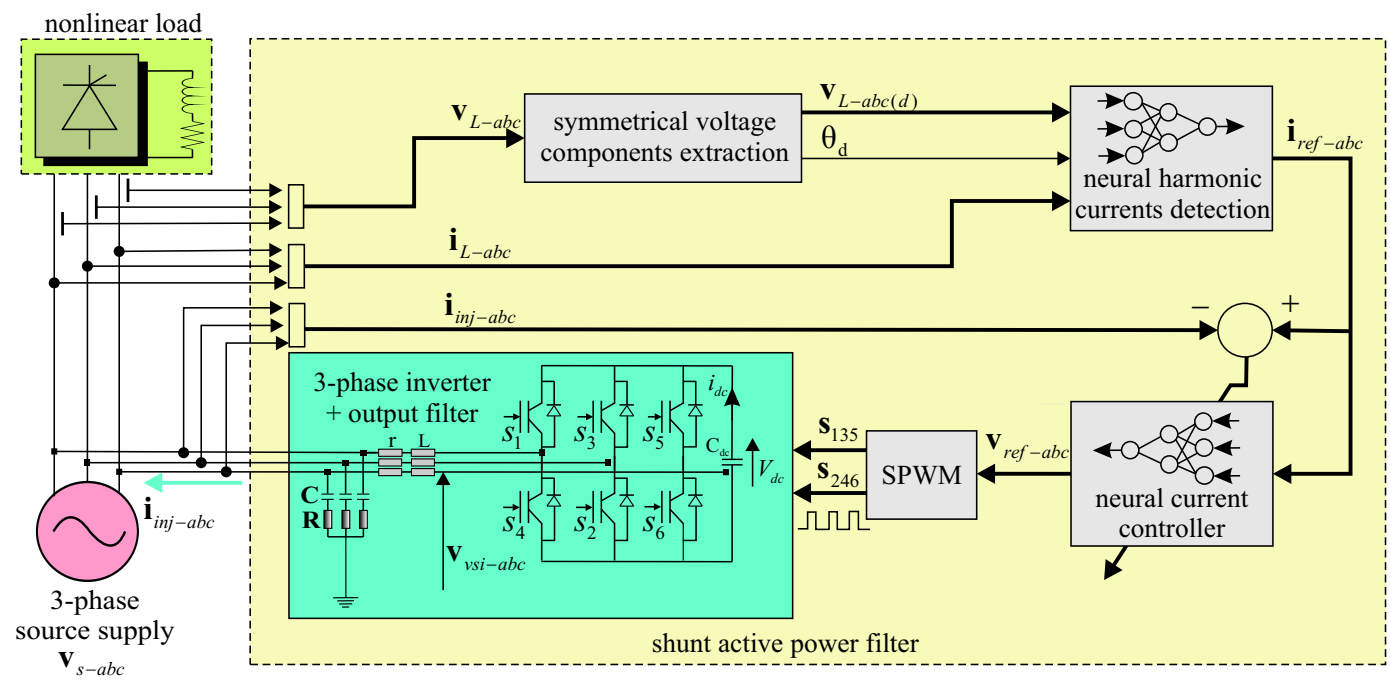

Figure 1: Block diagram of the shunt APF

IPT has since been enhanced and derived for systems under unbalanced conditions (Willems, 1996, Peng, 2001, Czarnecki, 2004, Herrera, Salmeron, and Kim, 2008).

The instantaneous active power is defined as the time rate of energy generation, transfer, or utilization. It is a physical quantity and satisfies the principle of conservation of energy. For a single-phase circuit, it is defined as the instantaneous product of the voltage and the current:

$$
p(t)=v(t) i(t) .
$$

For a circuit with $M$-phases, the instantaneous active power is expressed as (1) for each phase and the instantaneous total active power is the sum of the active powers of the individual phases:

$$
p(t)=\sum_{i=1}^{M} p_{i}(t)=\sum_{i=1}^{M} v_{i}(t) i_{i}(t) .
$$

The non-active power can be thought of as the useless power that cause losses, burdens and other harmful effects to power systems (Peng, 2001). The instantaneous non-active power or reactive power is called $q(t)$.

From the compensation standpoint, the goal of the IPT is to improve the power factor, to minimize power losses and to compensate for disturbances by identifying, measuring, and eliminating the useless power. As mentioned, the compensation is tackled with an APF. A shunt APF for minimizing the useless power and for compensating for the harmonic currents is shown by Fig. 1. Relevant currents and voltages at different points of its circuit are shown and enumerated thereafter: 
- $\mathbf{v}_{s-a b c}(t)$ is the three-phase voltage delivered by an AC source supply;

- $\mathbf{v}_{L-a b c}(t)$ is the three-phase voltage measured on the load;

- $\mathbf{i}_{L-a b c}(t)$ is the three-phase current measured on the load;

- $\mathbf{i}_{r e f-a b c}(t)$ is the identified harmonic currents of the three phases;

- $\mathbf{v}_{r e f-a b c}(t)$ is the control signal issued by a regulator to control the Voltage Source Inverter (VSI);

- $\mathbf{v}_{L-a b c(d)}(t)$ and $\theta_{d}(t)$ are respectively the vector of the instantaneous direct components and the instantaneous phase of the load voltage;

- $\mathbf{s}_{135}(t)=\left[\begin{array}{lll}s_{1} & s_{3} & s_{5}\end{array}\right]^{T}$ and $\mathbf{s}_{246}(t)=\overline{\mathbf{s}}_{135}(t)$, where each $s_{i} \in[1,0]$ with $i=$ $1,2 \ldots 6$, are the gates control signals defining the state of the IGBT (Insulated Gate Bipolar Transistor) gates, whether they conduct or not;

- $V_{d c}$ is the ideal supply voltage of the VSI;

- $\mathbf{v}_{v s i-a b c}(t)$ is the three-phase output voltage of the VSI;

- $\mathbf{i}_{\text {inj-abc }}(t)$ are three-phase compensating currents injected in the power distribution system.

Additionally, $T_{S}$ which is not represented on Fig. 1, is the sampling time of the numerical implementation of the APF control scheme. The continuous time is discretized, this allows to write $t=k T_{s}$ where $k$ represents the discrete time index.

One of the objective of the APF scheme is to characterize the harmonic terms generated by nonlinear loads. It is well-known that signals of three-phase systems can be represented by the sum of sine terms and by their direct, inverse and zero-sequence components. The three-phase voltages and currents measured on the nonlinear load can thus be respectively expressed by:

$$
\begin{aligned}
& \mathbf{v}_{L-a b c}(t)=\sum_{n=1}^{N}\left(\begin{array}{l}
\sqrt{2} V_{d n} \mathbf{C}_{32} \mathbf{P}\left(n \theta_{d}\right)\left[\begin{array}{ll}
1 & 0
\end{array}\right]^{T} \\
+\sqrt{2} V_{i n} \mathbf{C}_{32} \mathbf{P}\left(-n \theta_{i}\right)\left[\begin{array}{ll}
1 & 0
\end{array}\right]^{T} \\
+\sqrt{2} V_{0 n} \mathbf{C}_{31} \cos \left(n \theta_{0}\right)
\end{array}\right), \\
& \mathbf{i}_{L-a b c}(t)=\sum_{n=1}^{N}\left(\begin{array}{l}
\sqrt{2} I_{d n} \mathbf{C}_{32} \mathbf{P}\left(n \theta_{d}^{\prime}\right)\left[\begin{array}{ll}
1 & 0
\end{array}\right]^{T} \\
+\sqrt{2} I_{i n} \mathbf{C}_{32} \mathbf{P}\left(-n \theta_{i}^{\prime}\right)\left[\begin{array}{ll}
1 & 0
\end{array}\right]^{T} \\
+\sqrt{2} I_{0 n} \mathbf{C}_{31} \cos \left(n \theta_{0}^{\prime}\right)
\end{array}\right),
\end{aligned}
$$

where $n$ is the rank of the harmonic terms, $N$ represents the rank of the highest harmonic term taken into account, $\theta_{k}=\omega_{k} t+\phi_{k}$ and $\theta_{k}^{\prime}=\omega_{k} t+\phi_{k}^{\prime}$ with $k=d, i, o$ are the instantaneous phases with the phase angles $\phi_{k}$ and $\phi_{k}^{\prime}$ of respectively the voltages and the currents. The $d, i$ and $o$ indexes denote respectively the direct, 
inverse and zero-sequence components. In (3) and (4), $\mathbf{P}(\theta)$ is the matrix of the Park's transform:

$$
\mathbf{P}(\theta)=\left[\begin{array}{cc}
\cos \theta & -\sin \theta \\
\sin \theta & \cos \theta
\end{array}\right]
$$

and $\mathbf{C}_{32}$ and $\mathbf{C}_{31}$ are the matrices of the Clarke's transform

$$
\mathbf{C}_{31}=\left[\begin{array}{lll}
1 & 1 & 1
\end{array}\right]^{T}, \mathbf{C}_{32}^{T}=\left[\begin{array}{ccc}
1 & -1 / 2 & -1 / 2 \\
0 & \sqrt{3} / 2 & -\sqrt{3} / 2
\end{array}\right]
$$

which respect the following criteria (Akagi et al., 2007):

$$
\mathbf{C}_{32}^{T} \mathbf{C}_{31}=\left[\begin{array}{ll}
0 & 0
\end{array}\right]^{T}, \mathbf{C}_{32}^{T} \mathbf{C}_{32}=\frac{3}{2}\left[\begin{array}{ll}
1 & 0 \\
0 & 1
\end{array}\right]
$$

\subsection{The instantaneous power theory}

The IPT, or $\mathrm{p}-\mathrm{q}$ theory, is based on the instantaneous active power $p$, the reactive power $q$, and the zero-sequence power $p_{0}$ for defining systems under unbalanced conditions (Akagi et al., 2007). These powers are expressed as follow:

$$
\left[\begin{array}{c}
p \\
q \\
p_{0}
\end{array}\right]=\left[\begin{array}{ccc}
v_{\alpha} & v_{\beta} & 0 \\
v_{\beta} & -v_{\alpha} & 0 \\
0 & 0 & v_{0}
\end{array}\right]\left[\begin{array}{c}
i_{\alpha} \\
i_{\beta} \\
i_{0}
\end{array}\right]=\left[\begin{array}{c}
\bar{p}+\tilde{p} \\
\bar{q}+\tilde{q} \\
\bar{p}_{0}+\tilde{p}_{0}
\end{array}\right]
$$

with

$$
\begin{aligned}
& {\left[\begin{array}{lll}
v_{\alpha} & v_{\beta} & v_{0}
\end{array}\right]^{T}=\sqrt{\frac{2}{3}}\left[\begin{array}{ll}
\mathbf{C}_{32} & \frac{\mathbf{C}_{31}}{\sqrt{2}}
\end{array}\right]^{T} \mathbf{v}_{L-a b c},} \\
& {\left[\begin{array}{lll}
i_{\alpha} & i_{\beta} & i_{0}
\end{array}\right]^{T}=\sqrt{\frac{2}{3}}\left[\begin{array}{ll}
\mathbf{C}_{32} & \frac{\mathbf{C}_{31}}{\sqrt{2}}
\end{array}\right]^{T} \mathbf{i}_{L-a b c} .}
\end{aligned}
$$

As written in (8), the instantaneous power $p$ is always composed of an continuous part $\bar{p}$ and of a alternative part $\tilde{p}$. In the same way, $\bar{q}$ and $\tilde{q}$ are the continuous and the alternative parts of the instantaneous power $q, \bar{p}_{0}$ and $\tilde{p}_{0}$ the ones of $p_{0}$ respectively.

Developing the instantaneous active power $p$ from (8) leads to the following expression (with only the direct fundamental voltage component):

$$
\begin{aligned}
p= & \bar{p}+\sum_{n=2}^{N} p_{n-1} \cos \left((n-1) \omega t-\phi_{d 1}+\phi_{d n}^{\prime}\right) \\
& +\sum_{n=1}^{N} p_{n+1} \cos \left((n+1) \omega t+\phi_{d 1}+\phi_{i n}^{\prime}\right)
\end{aligned}
$$


International Journal of Emerging Electric Power Systems, Vol. 12 [2011], Iss. 5, Art. 1

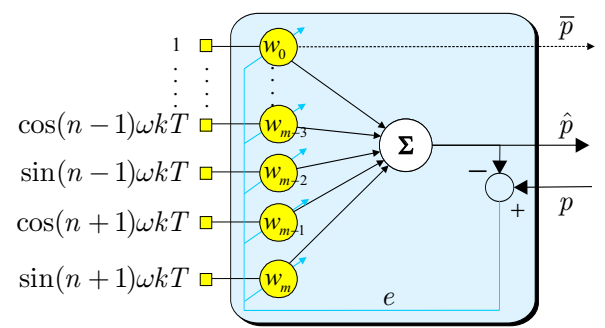

Figure 2: Adaline architecture for estimating the instantaneous active power $p$ according to the $\mathrm{p}-\mathrm{q}$ theory

where its continuous part is $\bar{p}=3 V_{d 1} I_{d 1} \cos \left(\phi_{d 1}-\phi_{d 1}^{\prime}\right)$ and with the coefficients $p_{n-1}=3 V_{d 1} I_{d n}$ and $p_{n+1}=3 V_{d 1} I_{i n}$. (11) can be rewritten with sums of vectors:

$$
\begin{gathered}
p=\bar{p}+\tilde{p}=\bar{p}+\sum_{n=2}^{N}\left(\left[\begin{array}{c}
\cos (n-1) \omega t \\
\sin (n-1) \omega t
\end{array}\right]^{T}\left[\begin{array}{c}
p_{n-1} \cos \left(\phi_{d 1}-\phi_{d n}^{\prime}\right) \\
p_{n-1} \sin \left(\phi_{d 1}-\phi_{d n}^{\prime}\right)
\end{array}\right]\right) \\
-\sum_{n=1}^{N}\left(\left[\begin{array}{c}
\cos (n+1) \omega t \\
\sin (n+1) \omega t
\end{array}\right]^{T}\left[\begin{array}{c}
p_{n+1} \cos \left(\phi_{d 1}+\phi_{i n}^{\prime}\right) \\
p_{n+1} \sin \left(\phi_{d 1}+\phi_{i n}^{\prime}\right)
\end{array}\right]\right) .
\end{gathered}
$$

In conventional compensation schemes, low-pass filters are used to extract $\bar{p}$. On the other side, it is possible to learn and to estimate the terms of $\tilde{p}$ in (12) by using Adalines (Ould Abdeslam et al., 2007). Compensating currents can be deduced from $\tilde{p}$ with $v_{\alpha \beta}^{2}=v_{\alpha}^{2}+v_{\beta}^{2}$ :

$$
\mathbf{i}_{r e f-a b c}=\frac{1}{v_{\alpha \beta}^{2}} \sqrt{\frac{2}{3}}\left[\begin{array}{ll}
\mathbf{C}_{32} & \frac{\mathbf{C}_{31}}{\sqrt{2}}
\end{array}\right]\left[\begin{array}{ccc}
v_{\alpha} & v_{\beta} & 0 \\
v_{\beta} & -v_{\beta} & 0 \\
0 & 0 & \frac{v_{\alpha \beta}^{2}}{v_{0}}
\end{array}\right]\left[\begin{array}{c}
\tilde{p} \\
q \\
p_{0}
\end{array}\right] .
$$

\subsection{Learning on-line linear sums of periodical signals}

The Adaline neural network was originally conceived and formulated for approximating linear combinations of scalar signals (Widrow and Walach, 1996). Based on the LMS (Least Mean Square) learning rule, this model represents a classic example of the simplest intelligent self-learning system that can adapt itself to achieve a given linear modeling task. The problem consists of finding a suitable set of weights such that the input-output behavior of the Adaline becomes close to a set of desired input-output data points linked with a linear relationship. The Adaline weights are 
solved using an iterative LMS algorithm in order to minimize the estimation error (Picton, 2000). The weights of the Adaline can be interpreted, giving thus a non negligible advantage to the Adaline over other ANNs.

The Adaline, whose architecture is represented on Fig. 2, represents a linear mapping between an input vector $\mathbf{x}(k)$ and a scalar output $y(k)$ at instant $k$, its output is thus:

$$
y(k)=\mathbf{x}^{T}(k) \mathbf{w}(k),
$$

and the weights are adjusted according to

$$
\mathbf{w}(k+1)=\mathbf{w}(k)+\eta \frac{e(k) \mathbf{x}(k)}{\mathbf{x}^{T}(k) \mathbf{x}(k)+\xi},
$$

where $\eta$ is a learning rate, $\xi$ is a small non-null positive constant and $e(k)=d(k)-$ $y(k)$ is the output error of the Adaline, i.e., the difference between $y(k)$ and a desired reference signal $d(k)$ measured on the system at each sampled time.

In the context of harmonic estimation, the underlying idea is to learn and approximate linear sums of sinusoidal signals with Adalines (Ould Abdeslam et al., 2007). Appropriate sinusoidal signals that are representative of possible harmonic terms are synthesized and used as inputs. Then, the Adaline compares its output to a measured variable and adjusts its weights in consequence. The weights of the Adaline are thus enforced to converge toward values which represent the amplitudes of the sinusoidal signals. Applied to a power system, the final weights are representative of the amplitudes of the real harmonic terms.

For example, one Adaline can be used to estimate the decomposition of $p$ given in (12). Therefore, the following generic input vector is created to fulfill the expression of $p$ with $n=2$ to $N$ (see also Fig. 2):

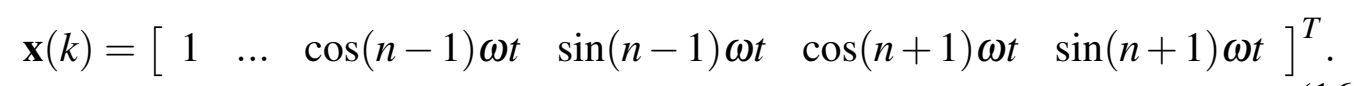

After learning, the weights w of the Adaline converge to:

$$
\mathbf{w}^{*}(k) \underset{k \rightarrow \infty}{\longrightarrow}\left[\begin{array}{llll}
\bar{p} & \ldots & \mathbf{p}_{N-1}^{T} & \mathbf{p}_{N+1}^{T}
\end{array}\right]^{T}
$$

with

$$
\mathbf{p}_{N-1}=\left[\begin{array}{c}
p_{n-1} \cos \left(\phi_{d 1}-\phi_{d n}^{\prime}\right) \\
p_{n-1} \sin \left(\phi_{d 1}-\phi_{d n}^{\prime}\right)
\end{array}\right], \mathbf{p}_{N+1}=\left[\begin{array}{c}
p_{n+1} \cos \left(\phi_{d 1}+\phi_{i n}^{\prime}\right) \\
p_{n+1} \sin \left(\phi_{d 1}+\phi_{i n}^{\prime}\right)
\end{array}\right]
$$

It can be seen that the weights $\mathbf{w}^{*}(k)$ contain continuous terms corresponding to the amplitude of each power harmonic and that its first element is the continuous part of $p$. The output of the Adaline is $\hat{p}$, the estimation of the instantaneous power 
$p$, and its desired value is the power $p$ recovered from the measured currents and voltages. Two other Adalines are used to estimate the instantaneous powers $q$ and $p_{0}$ of (8). This has been proposed in (Ould Abdeslam et al., 2007) to identify and to compensate in real-time for the most common harmonic terms in power distribution systems which are of rank $n=5,7,11,13,17,19,23,25 \ldots$ i.e., by using the terms $\cos 6 j \omega t$ and $\sin 6 j \omega t$ with $j=1,2,3 \ldots$ for estimating the powers.

The principle of formalizing expressions of instantaneous variables by mean of sums of sinusoidal terms for estimating their amplitudes with Adalines has been applied to other types of variables. More specifically, the following section proposes to learn modified p-q powers, active currents and synchronized currents. In each decomposition learned by Adalines, the resulting weights allow to recover the amplitudes of the harmonic terms of either instantaneous powers or currents.

\section{Original neural schemes for harmonic currents iden- tification}

Three neural schemes for harmonic currents identification are developed. The first method is based on the modified instantaneous p-q theory, the second one is based on optimal active currents and the third method is based on synchronized currents. Each one allows to clearly identify the harmonic terms and thus to compensate for them with different objectives: full compensation, selective harmonic compensation, power factor correction, unbalance correction, and power flow control.

In the case of unbalanced nonlinear loads, a symmetrical component extraction algorithm is necessary in order to estimate the direct voltage components, i.e., $\mathbf{v}_{L-a b c(d)}(t)$. A Phase Locked Loop (PLL) can be used; we adopted the neural approach developed in (Flieller, Ould Abdeslam, Wira, and Mercklé, 2009).

\subsection{Neural method based on the modified p-q theory}

Most APF devices use the instantaneous powers defined by the well-known p-q method (Akagi, 1996) which is not appropriate in all cases. Extensions and alternative methods have been proposed since, like the modified p-q method which uses at the same time the active, reactive and zero-sequence instantaneous powers (Akagi et al., 2007). Under balanced conditions, the modified and the original p-q methods use the same expression of the continuous parts of the powers. This is not the case with unbalanced nonlinear loads. The instantaneous power $q$ can be separated into two parts, $q_{\alpha}$ and $q_{\beta}$. Their resulting expressions are learned by Adalines (Nguyen, Wira, Ould Abdeslam, and Flieller, 2009) as will be explained thereafter. 
Nguyen et al.: Harmonics Identification with ANNs : Application to APF

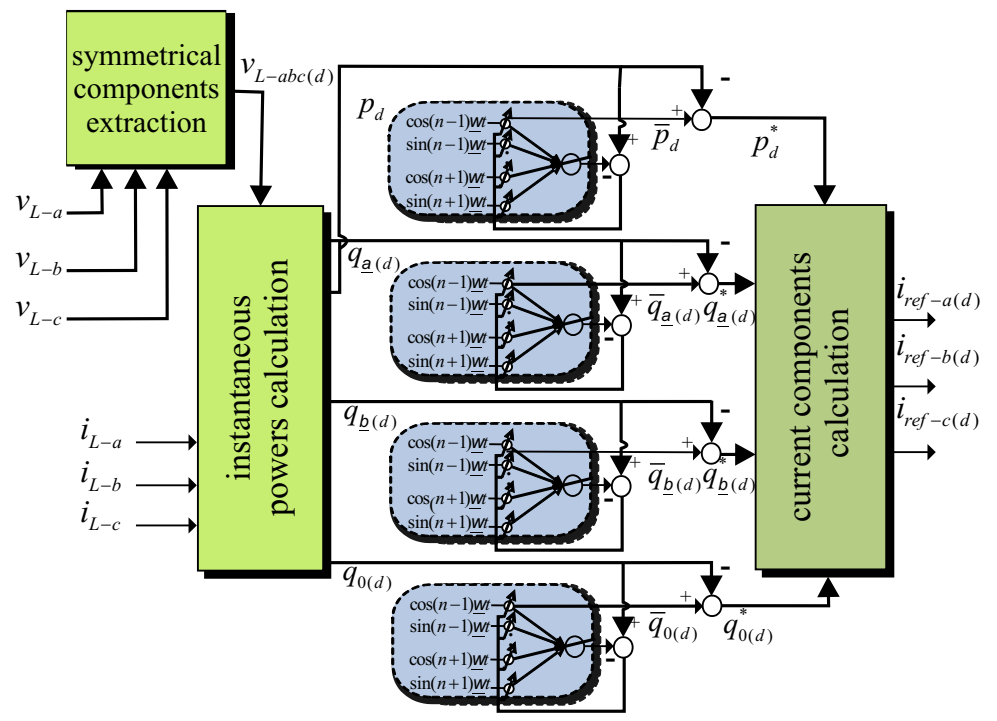

Figure 3: Adaline-based modified p-q method

Under unbalanced conditions, the modified p-q theory is based on the following expressions of the powers:

$$
\left[\begin{array}{c}
p \\
q_{\alpha} \\
q_{\beta} \\
q_{0}
\end{array}\right]=\left[\begin{array}{ccc}
v_{\alpha} & v_{\beta} & v_{0} \\
0 & -v_{0} & v_{\beta} \\
v_{0} & 0 & -v_{\alpha} \\
-v_{\beta} & v_{\alpha} & 0
\end{array}\right]\left[\begin{array}{c}
i_{\alpha} \\
i_{\beta} \\
i_{0}
\end{array}\right],
$$

where $v_{\alpha}, v_{\beta}, v_{0}, i_{\alpha}, i_{\beta}$ and $i_{0}$ come from (9) and (10).

The active power $p$ in (19) can be detailed by:

$$
\begin{aligned}
p & =\bar{p}+\tilde{p}, \\
\bar{p} & =\sum_{k=d, i, 0} \sum_{n=1}^{N} 3 V_{k n} I_{k n} \cos \left(\phi_{k n}-\phi_{k n}^{\prime}\right), \\
\tilde{p} & =\sum_{k=d, i, 0} \sum_{m \neq n}^{N} \sum_{n=1}^{N} 3 V_{k m} I_{k n} \cos \left(\theta_{k m}-\theta^{\prime}{ }_{k n}\right), \\
& +\sum_{k=d, i, 0} \sum_{m=1}^{N} \sum_{n=1}^{N}-3 V_{k m} I_{k^{*} n} \cos \left(\theta_{k m}+\theta^{\prime}{ }_{k^{*} n}\right) \text { with } k^{*}=\left\{\begin{array}{cc}
i & \text { if } k=d \\
d & \text { if } k=i \\
0 & \text { if } k=0
\end{array} .\right.
\end{aligned}
$$

This approach separates the reactive power $q$ into three components, i.e., the $\alpha-\beta$ components and the zero-sequence component. The reactive power along the $\alpha$ 
International Journal of Emerging Electric Power Systems, Vol. 12 [2011], Iss. 5, Art. 1

axis is

$$
\begin{aligned}
q_{\alpha} & =-v_{0} i_{\beta}+v_{\beta} i_{0}=\bar{q}_{\alpha}+\tilde{q}_{\alpha}, \\
\bar{q}_{\alpha} & =\sum_{k=d, i} \sum_{n=1}^{N} a \frac{3 \sqrt{2}}{2}\left[\begin{array}{c}
V_{0 n} I_{k n} \\
-V_{k n} I_{0 n}
\end{array}\right]^{T}\left[\begin{array}{c}
\sin \left(\phi_{o n}-\phi_{k n}^{\prime}\right) \\
-\sin \left(\phi_{k n}-\phi_{o n}^{\prime}\right)
\end{array}\right], \\
\tilde{q}_{\alpha} & =\sum_{k=d, i} \sum_{m \neq n}^{N} \sum_{n=1}^{N} a \frac{3 \sqrt{2}}{2} \mathbf{A}\left[\begin{array}{c}
\sin \left(\theta_{o m}-\theta^{\prime}{ }_{k n}\right) \\
-\sin \left(\theta_{k m}-\theta^{\prime}{ }_{o n}\right)
\end{array}\right] \\
& +\sum_{k=d, i m=1} \sum_{n=1}^{N} \sum_{n=1}^{N} a \frac{3 \sqrt{2}}{2} \mathbf{A}\left[\begin{array}{c}
\sin \left(\theta_{o m}+\theta^{\prime}{ }_{k n}\right) \\
\sin \left(\theta_{k m}+\theta^{\prime}{ }_{o n}\right)
\end{array}\right] \text { with } a=\left\{\begin{array}{cc}
1 & \text { if } k=d \\
-1 & \text { if } k=i
\end{array},\right.
\end{aligned}
$$

and where $\mathbf{A}=\left[\begin{array}{ll}A_{1} & A_{2}\end{array}\right]=\left[\begin{array}{ll}V_{0 m} I_{k n} & -V_{k m} I_{0 n}\end{array}\right]$.

The reactive power along the $\beta$ axis is obtained in the same way but with an angle shifted of $\frac{\pi}{2}$. The sine terms of (24) and (25) can thus be replaced by cosine terms for defining $\bar{q}_{\beta}$ and $\tilde{q}_{\beta}$. The power $q_{0}$ is determined in the same way as $q$ in the original p-q method:

$$
\begin{aligned}
q_{0} & =\bar{q}_{0}+\tilde{q}_{0}, \\
\bar{q}_{0} & =\sum_{k=d, i} \sum_{n=1}^{N} 3 a V_{k n} I_{k n} \sin \left(\phi_{k n}-\phi_{k n}^{\prime}\right), \\
\tilde{q}_{0} & =\sum_{k=d, i} \sum_{m \neq n}^{N} \sum_{n=1}^{N}-3 a V_{k m} I_{k n} \sin \left(\theta_{k m}-\theta^{\prime}{ }_{k n}\right) \\
& +\sum_{k=d, i} \sum_{m=1}^{N} \sum_{n=1}^{N} 3 a V_{k m} I_{k^{*} n} \sin \left(\theta_{k m}+\theta^{\prime}{ }_{k^{*} n}\right) .
\end{aligned}
$$

Expressions (20) to (28) determine instantaneous powers in terms of linear weighted sums of sinusoidal signals. Indeed, $p, q_{\alpha}$ and $q_{0}$ respectively given by (20), (23) and (26), and $q_{\beta}$ which can be deduced from $q_{\alpha}$ by replacing the sine terms by cosine terms in (23), are sums of sinusoidal signals weighted by coefficients. For example, $p$ can be written in the form of (14) with:

$$
\mathbf{x}=\left[\begin{array}{c}
\cos (n-1) \omega t \\
\sin (n-1) \omega t \\
\cos (n+1) \omega t \\
\sin (n+1) \omega t
\end{array}\right], \mathbf{w}=\left[\begin{array}{c}
3 V_{d 1} I_{d n} \cos \left(\phi_{d 1}-\phi_{d n}^{\prime}\right) \\
3 V_{d 1} I_{d n} \sin \left(\phi_{d 1}-\phi_{d n}^{\prime}\right) \\
-3 V_{d 1} I_{i n} \cos \left(\phi_{d 1}+\phi_{i n}^{\prime}\right) \\
3 V_{d 1} I_{i n} \sin \left(\phi_{d 1}+\phi_{i n}^{\prime}\right)
\end{array}\right]
$$

with $n=1, \ldots N . \mathbf{x}$ is the input vector of the Adaline which contains synthesized signals representing the $N$ harmonic terms. $\mathbf{w}$ is the weights vector of the Adaline. 
With these vectors, the output of the Adaline given by (14) provides an estimation of $p$ which is on-line compared to measured values. The iterative LMS learning rule makes the weights to converge toward the appropriate coefficients. The weights of the Adaline therefore represent the amplitudes of the harmonic terms composing $p$. The first weight is associated to an input which is the fundamental term and therefore represents the continuous active power produced by the direct fundamental currents. Fig. 3 illustrates the principle of estimating the harmonic terms of $p$. The harmonic terms of the three other reactive powers are estimated according to the same principle.

Under unbalanced conditions, this approach must be completed by a symmetrical voltage components extraction algorithm. This can be achieved by a conventional PLL or a neural dedicated scheme (Flieller et al., 2009). If the voltages system is unbalanced, the continuous parts of the powers $p, q_{\alpha}, q_{\beta}$ and $q_{0}$ consist of three components from the direct, inverse and zero-sequence system. As a consequence, the reference currents from (30) will not be able to compensate for the whole imbalance. These currents will only be able to maintain a constant active power. Generally, an APF ensures a constant active power in the distribution system but also a sinusoidal waveforms of the source currents.

This compensation approach allows to handle not only four-wire but also three-wire systems. According to the compensating objective, the instantaneous powers can be used to elaborate the reference currents to be injected in the power distribution system:

$$
\mathbf{i}_{r e f-a b c}=\frac{1}{v_{\alpha \beta 0}^{2}} \sqrt{\frac{2}{3}}\left[\begin{array}{ll}
\mathbf{C}_{32} & \mathbf{C}_{31} \\
\sqrt{2}
\end{array}\right]\left[\begin{array}{cccc}
v_{\alpha} & 0 & v_{0} & -v_{\beta} \\
v_{\beta} & -v_{0} & 0 & v_{\alpha} \\
v_{0} & v_{\beta} & -v_{\alpha} & 0
\end{array}\right]\left[\begin{array}{c}
p \\
q_{\alpha} \\
q_{\beta} \\
q_{0}
\end{array}\right]
$$

where $v_{\alpha \beta 0}^{2}=v_{\alpha}^{2}+v_{\beta}^{2}+v_{0}^{2}$. Even if the zero-sequence voltage is null, the modified $\mathrm{p}$-q method considers the zero-sequence current $i_{0}$ as a reactive current because of its contribution to the reactive powers $q_{\alpha}$ and $q_{\beta}$ as mentioned in (19). A compensation scheme without any energy storage unit can be used to fully cancel $i_{0}$ and the harmonic currents if $v_{0}=0$. It can be noticed that $i_{0}$ is not canceled if the reactive powers $q_{\alpha}$ and $q_{\beta}$ are not taken into account in the right hand-side of (30), i.e., if they are replaced by 0 .

\subsection{Neural method based on active currents}

The instantaneous reactive current is a component which does not contribute to the active power (Akagi et al., 2007). This current contributes to the amplitude 
International Journal of Emerging Electric Power Systems, Vol. 12 [2011], Iss. 5, Art. 1

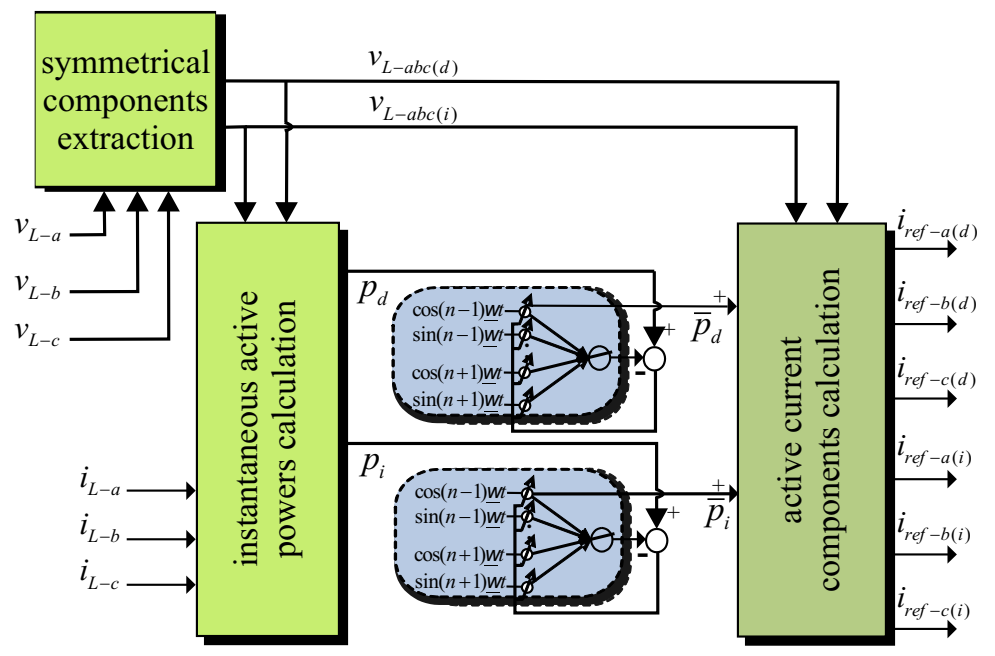

Figure 4: Adaline-based active currents method

of the total current but increases the losses. This current can be estimated by different ways. We propose to use the Lagrange multiplier method (Luenberger and Ye, 2008) to calculate the active currents from instantaneous powers estimated by Adalines (Nguyen et al., 2009).

In a power distribution system with $l+1$ wires, i.e., $l$ phases and a neutral, the voltages and currents of the nonlinear load can be respectively defined by the following vectors:

$$
\begin{aligned}
\mathbf{v} & =\left[\begin{array}{llll}
v_{1} & v_{2} & \ldots & v_{l}
\end{array}\right]^{T}, \\
\mathbf{i} & =\left[\begin{array}{llll}
i_{1} & i_{2} & \ldots & i_{l}
\end{array}\right]^{T} .
\end{aligned}
$$

The active currents method estimates the active part of the load currents under the constraint that these currents produce the instantaneous active power $p$ (Akagi et al., 2007) by the following expression:

$$
p=\mathbf{v}^{T} \mathbf{i}
$$

Two optimization strategies are proposed to calculate the active currents minimizing the losses of the network with a given instantaneous power $p$.

\subsubsection{Optimization on $l$ lines with $i_{0}=0$}

The optimization can be achieved by considering $l$ lines. In this case, the neutral current have to be equal to zero at the source-side and this means: 


$$
\mathbf{u}_{1}^{T} \mathbf{i}=0
$$

with $\mathbf{u}_{1}=\left[\begin{array}{llll}1 & 1 & \cdots & 1\end{array}\right]^{T}$.

There is an infinite number of solutions, i.e., of line currents, which satisfy at once equations (33) and (34). However, a unique solution can be determined by introducing the ohmic losses

$$
g(\mathbf{i})=\frac{1}{2} \mathbf{i}^{T} \mathbf{i}
$$

and the objective function $L_{1}$ can thus be used:

$$
L_{1}=g(\mathbf{i})+\lambda_{1}\left(p-\mathbf{v}^{T} \mathbf{i}\right)+\lambda_{2} \mathbf{u}_{1}^{T} \mathbf{i}
$$

We can write (37) by taking the partial derivatives of $L_{1}$ according to the each phase current and equating them to zero:

$$
\mathbf{i}=\lambda_{1} \mathbf{v}-\lambda_{2} \mathbf{u}_{1}
$$

Replacing (37) into (33) leads to:

$$
p=\mathbf{v}^{T} \mathbf{i}=\mathbf{v}^{T}\left(\lambda_{1} \mathbf{v}-\lambda_{2} \mathbf{u}_{1}\right)=\lambda_{1} \mathbf{v}^{T} \mathbf{v}-\lambda_{2} \mathbf{v}^{T} \mathbf{u}_{1} .
$$

Replacing (37) into (34) gives:

$$
\mathbf{u}_{1}^{T} \mathbf{i}=\mathbf{u}_{1}^{T}\left(\lambda_{1} \mathbf{v}-\lambda_{2} \mathbf{u}_{1}\right)=\lambda_{1} \mathbf{u}_{1}^{T} \mathbf{v}-l \lambda_{2}=0
$$

The following two Lagrangian multipliers can be calculated from (38) and (39):

$$
\begin{aligned}
& \lambda_{1}=\frac{l}{l \mathbf{v}^{T} \mathbf{v}-\left(\mathbf{u}_{1}^{T} \mathbf{v}\right)^{2}} p, \\
& \lambda_{2}=\frac{\mathbf{u}_{1}^{T} \mathbf{v}}{l \mathbf{v}^{T} \mathbf{v}-\left(\mathbf{u}_{1}{ }^{T} \mathbf{v}\right)^{2}} p .
\end{aligned}
$$

Finally, the line currents can be expressed with $\lambda_{1}$ et $\lambda_{2}$ by:

$$
\mathbf{i}_{\text {opt }(l)}=l \frac{\mathbf{v}}{l \mathbf{v}^{T} \mathbf{v}-\left(\mathbf{u}_{1}^{T} \mathbf{v}\right)^{2}} p-\frac{\mathbf{u}_{1}^{T} \mathbf{v u}_{1}}{l \mathbf{v}^{T} \mathbf{v}-\left(\mathbf{u}_{1}{ }^{T} \mathbf{v}\right)^{2}} p=\frac{l\left(\mathbf{v}-\frac{1}{l} \mathbf{u}_{1}^{T} \mathbf{v u}_{1}\right)}{l \mathbf{v}^{T} \mathbf{v}-\left(\mathbf{u}_{1}^{T} \mathbf{v}\right)^{2}} p .
$$

The currents defined by (42) release instantaneously a constant active power and minimize the ohmic losses in the $l$ lines of the power system. If a neutral current is allowed, i.e. $i_{0} \neq 0$, then an alternative strategy is proposed to minimize the Joule losses on $l+1$ lines. 


\subsubsection{Optimization on $l+1$ lines with $i_{0} \neq 0$}

In the case of $l+1$ lines, the objective function is defined by introducing the ohmic losses on the $l$ phases and the neutral line according to:

$$
L_{2}=g(\mathbf{i})+\frac{1}{2}\left(\mathbf{u}_{1}{ }^{T} \mathbf{i}\right)+\lambda_{3}\left(p-\mathbf{v}^{T} \mathbf{i}\right) .
$$

With the same developments as in the previous case, we establish:

$$
\mathbf{i}+\mathbf{u}_{1}{ }^{T} \mathbf{i}=\underbrace{\left[\begin{array}{ccccc}
2 & 1 & 1 & \ldots & 1 \\
1 & 2 & 1 & \ldots & 1 \\
1 & 1 & 2 & \ldots & 1 \\
\vdots & & & & \\
1 & 1 & 1 & \ldots & 2
\end{array}\right]}_{l \times l} \mathbf{i}=\lambda_{3} \mathbf{v}
$$

and:

$$
\mathbf{i}=\frac{\lambda_{3}}{(l+1)} \underbrace{\left[\begin{array}{ccccc}
l & -1 & -1 & \ldots & -1 \\
-1 & l & -1 & \ldots & -1 \\
-1 & -1 & l & \ldots & -1 \\
\vdots & & & & \\
-1 & -1 & -1 & \ldots & l
\end{array}\right]}_{\mathbf{A}} \mathbf{v} .
$$

Replacing (45) into (33) leads to:

$$
p=\frac{\lambda_{3}}{(l+1)} \mathbf{v}^{T} \mathbf{A} \mathbf{v},
$$

with:

$$
\begin{aligned}
\mathbf{v}^{T} \mathbf{A} \mathbf{v} & =l \mathbf{v}^{T} \mathbf{v}-2 \sum_{k=1}^{l} \sum_{j \neq k ; j=1}^{l} v_{k} v_{j} \\
& =l \mathbf{v}^{T} \mathbf{v}-\left(\left(\mathbf{u}_{1}^{T} \mathbf{v}\right)^{2}-\mathbf{v}^{T} \mathbf{v}\right)=(l+1) \mathbf{v}^{T} \mathbf{v}-\left(\mathbf{u}_{1}^{T} \mathbf{v}\right)^{2} .
\end{aligned}
$$

The Lagrangian $\lambda_{3}$ is thus deduced:

$$
\lambda_{3}=\frac{(l+1) p}{(l+1) \mathbf{v}^{T} \mathbf{v}-\left(\mathbf{u}_{1}^{T} \mathbf{v}\right)^{2}},
$$


and the line currents are obtained by substituting $\lambda_{3}$ into (45):

$$
\mathbf{i}_{\mathrm{opt}(l+1)}=\frac{(l+1) \mathbf{v}-\mathbf{u}_{1}^{T} \mathbf{v} \mathbf{u}_{1}}{(l+1) \mathbf{v}^{T} \mathbf{v}-\left(\mathbf{u}_{1}^{T} \mathbf{v}\right)^{2}} p .
$$

Expression (49) provides the active currents that optimize the transmission losses in $(l+1)$-wire systems. A comparative study shows that in $l$-wire systems, the transmission losses are equivalent if the compensation is achieved by the p-q method or by the active currents (Malengret and Gaunt, 2008). On the other side, the losses are lower with the active currents method than with the p-q method in $(l+1)$-wire systems.

It can be seen that the optimal current $\mathbf{i}_{\text {opt }}$, which is $\mathbf{i}_{\text {opt }(l)}$ or $\mathbf{i}_{\text {opt }(l+1)}$ according to the considered strategy, is obtained from the instantaneous powers and in particular from (33). This power is learned with two Adalines, one for estimating $p_{d}$, the active power of the direct voltage and current components and one for estimating $p_{i}$, the active power of the inverse voltage and current components.

A compensating scheme for a three-phase nonlinear load described by $\mathbf{i}=$ $\mathbf{i}_{L-a b c}$ uses the following reference currents:

$$
\mathbf{i}_{r e f-a b c}=\mathbf{i}_{L-a b c}-\mathbf{i}_{\mathrm{opt}} .
$$

It is illustrated by Fig. 4. The active currents are sinusoidal signals obtained from the amplitude of the active currents synchronized with the direct voltage components. As a consequence, the power factor is corrected and achieved near to unity. The compensation of the reactive power can be considered as a part of the harmonics compensation strategy.

\subsection{Neural synchronized method}

The neural synchronized method implicitly works in a reference frame which is synchronized with the direct voltage components (Nguyen, Ould Abdeslam, Wira, Flieller, and Mercklé, 2008). The amplitude of the fundamental current $I_{1}$ and the phase-shift angle of the positive voltage are extracted from a decomposition of the currents proposed in (Moran, Dixon, and Wallace, 1995). This decomposition allows to formulate the harmonics in a reference frame obtained by multiplying the load current indifferently by $\sin \theta_{d}$ or by $\cos \theta_{d}$ issued from a symmetrical components extraction algorithm. This reference frame can be considered as representing a virtual power synchronized with the source voltage of the system. The principle of this method is described by Fig. 5. 
International Journal of Emerging Electric Power Systems, Vol. 12 [2011], Iss. 5, Art. 1

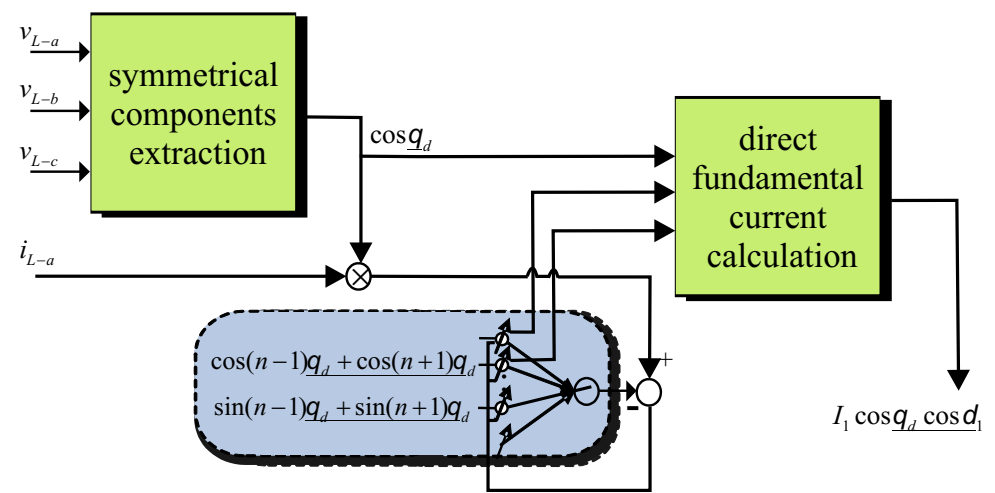

Figure 5: Principle of the Adaline-based synchronous method for one phase

Therefore, the three-phase voltage of (3) with only the direct components can be written as:

$$
\begin{aligned}
\mathbf{v}_{L-a b c}(t) & =\sum_{n=1}^{N} \sqrt{2} V_{n}\left[\begin{array}{c}
\cos (n(\omega t+\varphi)) \\
\cos (n(\omega t-2 \pi / 3+\varphi)) \\
\cos (n(\omega t+2 \pi / 3+\varphi))
\end{array}\right] \\
= & \sum_{n=1}^{N} \sqrt{2} V_{n}\left[\begin{array}{c}
\cos \left(n \theta_{d}\right) \\
\cos \left(n \theta_{d}-n 2 \pi / 3\right) \\
\cos \left(n \theta_{d}+n 2 \pi / 3\right)
\end{array}\right]
\end{aligned}
$$

with $\theta_{d}=\omega t+\varphi$. A symmetrical components extraction algorithm or a PLL allows to recover the direct voltage components which are without harmonic terms:

$$
\mathbf{v}_{L-a b c(d)}(t)=\sqrt{2} V\left[\begin{array}{c}
\cos \left(\theta_{d}\right) \\
\cos \left(\theta_{d}-2 \pi / 3\right) \\
\cos \left(\theta_{d}+2 \pi / 3\right)
\end{array}\right] .
$$

Here, the objective of the compensation is to recover sinusoidal current waveforms which are in phase with the direct voltage components. As a consequence, the total free and available power transmitted from the source to the load will be at its maximum. The three-phase current defined by (4) with only the direct components can be rewritten by:

$$
\mathbf{i}_{L-a b c}(t)=\sum_{n=1}^{N} \sqrt{2} I_{n}\left[\begin{array}{c}
\cos \left(n \theta_{d}+\delta_{n}\right) \\
\cos \left(n \theta_{d}-2 n \pi / 3+\delta_{n}\right) \\
\cos \left(n \theta_{d}+2 \pi / 3+\delta_{n}\right)
\end{array}\right]
$$

with $\delta_{n}$ the phase of harmonic term of rank $n$ expressed between the voltage and the current components. Multiplying the load current of the first phase by $\cos \theta_{d}$ issued 
from the symmetrical components extraction algorithm gives:

$$
\begin{aligned}
& i_{L-a}(t) \cos \theta_{d}= \\
& \quad \sum_{n=1}^{N} \frac{\sqrt{2} I_{n}}{2}\left\{\cos \left((n+1) \theta_{d}+\delta_{n}\right)+\cos \left((n-1) \theta_{d}+\delta_{n}\right)\right\} \\
& =\sum_{n=1}^{N} \frac{\sqrt{2} I_{n}}{2}\left\{\cos (n+1) \theta_{d} \cdot \cos \delta_{n}-\sin (n+1) \theta_{d} \cdot \sin \delta_{n}\right\} \\
& +\sum_{n=1}^{N} \frac{\sqrt{2} I_{n}}{2}\left\{\cos (n-1) \theta_{d} \cdot \cos \delta_{n}-\sin (n-1) \theta_{d} \cdot \sin \delta_{n}\right\}
\end{aligned}
$$

This expression is a weighted sum of harmonic terms which can be decomposed with the following generic vectors:

$$
\begin{gathered}
\mathbf{x}=\left[\begin{array}{cc}
\cos (n-1) \theta_{d}+\cos (n+1) \theta_{d} & \sin (n-1) \theta_{d}+\sin (n+1) \theta_{d}
\end{array}\right]^{T} \\
\mathbf{w}=\left[\begin{array}{cc}
\frac{I_{n} \cos \delta_{n}}{\sqrt{2}} & -\frac{I_{n} \sin \delta_{n}}{\sqrt{2}}
\end{array}\right]^{T}
\end{gathered}
$$

where $n=1, \ldots N$. Expression (54) is learned with one Adaline using $\mathbf{x}$ as an input and $i_{L-a}(t) \cos \left(\theta_{d}\right)$ its desired output. $\theta_{d}$ is estimated by a symmetrical components extraction algorithm. The learning therefore enforces the weights vector to converge toward $\mathbf{w}$ of (56). The values of the two first weights $\mathbf{w}^{* 1}=\frac{I_{1} \cos \delta_{1}}{\sqrt{2}}$ and $\mathbf{w}^{* 2}=-\frac{I_{1} \sin \delta_{1}}{\sqrt{2}}$ are thus associated to the direct fundamental sinusoidal term of the load currents and can be used to calculate $I_{1}$ and $\delta_{1}$ :

$$
\begin{gathered}
I_{1}=\sqrt{2\left(\mathbf{w}^{* 1}\right)^{2}+2\left(\mathbf{w}^{* 2}\right)^{2}}, \\
\delta_{1}=\arctan \frac{-\mathbf{w}^{* 2}}{\mathbf{w}^{* 1}} .
\end{gathered}
$$

In the same way, it is possible to calculate the amplitude and phase of each harmonic term, $I_{n}$ and $\delta_{n}$, by using the corresponding Adaline weights $\mathbf{w}^{*(2 n-1)}=\frac{I_{n} \cos \delta_{n}}{\sqrt{2}}$ and $\mathbf{w}^{*(2 n)}=\frac{I_{n} \sin \delta_{n}}{\sqrt{2}}$. Each harmonic term can thus be taken into account individually. Compensating only for the specific harmonic of rank $n>1$ requires the following reference current for the first phase:

$$
i_{r e f-a}=I_{n} \cos \left(\theta_{d n}+\delta_{n}\right)+I_{1} \sin \delta_{1} \cos \theta_{d} .
$$

The reference current for compensating for of all harmonics at once can be calculated by the sum of the higher-order harmonics or more simply by:

$$
i_{r e f-a}=i_{L-a}-I_{1} \cos \delta_{1} \cos \theta_{d} .
$$


In (60), the current $I_{1} \cos \delta_{1} \cos \theta_{d}$ is an active current in phase with the direct voltage component and represents the active fundamental term of the load current. As a consequence, a compensation scheme with reference currents provided by (59) or by (60) inherently maintains the power factor to unity.

\subsection{Discussion}

The neural approaches proposed for the identification of the harmonic currents are all based on instantaneous powers estimated by Adalines. One is based on the modified p-q powers, one on the active powers $p_{d}$ and $p_{i}$, and one on a virtual power defined by the measured load currents and a recovered voltage $\cos \theta_{d}$.

Their characteristics are presented and compared to a neural scheme based on the conventional p-q theory in Table 1 . The expressions for calculating the reference currents are recapitulated and completed by the variables required according to the compensating objectives. The number of Adalines involved in each neural scheme is specified, their compatibility with single or three phase power systems and a rough estimation of the computational costs are provided.

The proposed harmonic currents identification methods can all be used in 3wire or 4-wire three-phase power systems. If the voltage components are balanced, all the proposed neural schemes demonstrate a quite similar behavior. On the other hand, with unbalanced voltage components, their behavior differs with the number of wires. In 3-wire three-phase power systems, the neural method based on active currents optimized for 3 phases leads to a null homopolar current after compensation. This is also the case of the method based on the conventional p-q powers because it uses the same expression of the power in this particular case. In 4-wire three-phase power systems, the neural method based on the modified p-q powers results in highest Joule losses. The most reduced losses are obtained with the neural method based on active currents when they are optimized for 4 phases. These consideration are important, especially for controlling synchronous machines and maintaining at the same time a constant torque and minimum Joules losses with a non sinusoidal electromotive force.

The scheme based on the modified p-q powers is the most computationally extensive method with 4 Adalines, against 2 Adalines for the neural method based on the conventional p-q powers and only one for the two other methods. The simplest implementation is achieved with the neural synchronized method. 
Nguyen et al.: Harmonics Identification with ANNs : Application to APF

\section{Evaluation of the proposed neural harmonic iden- tification schemes by experimental tests}

The precision and the robustness of the proposed Adaline-based harmonic identification schemes are evaluated with real-time experiments. The schemes are inserted in a shunt APF for compensating harmonics generated by a nonlinear and unbalanced load.

\subsection{Experimental setup}

The experimental set-up is a three-phase sinusoidal electrical grid at $130 \mathrm{~V} \mathrm{rms}$ and $50 \mathrm{~Hz}$ with a $1 \mathrm{kVA}$ nonlinear load. This load is composed of a diode bridge supplying a highly inductive load and a linear resistive load and generates higherorder harmonics up to rank 30. The nonlinear load fluctuates and changes therefore the harmonic content of the currents and the unbalance. A dSPACE-based APF prototype is inserted for identifying the harmonics and improving the power quality under various operating conditions. This system is represented by Fig. 6 .

The control of the APF is implemented as a stand-alone process on a dSPACE board with a sampling period $T_{S}=0.6 \mathrm{~ms}$. Mainly, the neural harmonics identification schemes are hosted by this high-speed DSP board. Once calculated, compensating currents are generated by a three-phase VSI. This inverter is connected to the grid by a filtering inductance $L=3.8 \mathrm{mH}$ and a resistance $r=2.5 \Omega$ and the current control is ensured by a neural controller (Ould Abdeslam et al., 2007).

\subsection{Compensating for all harmonic currents}

The compensation of the harmonics is achieved by the proposed neural schemes. Their performances are also compared to the performance of their original methods.

Table 1: Characteristics of the neural harmonics identification schemes

\begin{tabular}{|c|c|c|c|c|c|c|c|}
\hline $\begin{array}{l}\text { identification } \\
\text { method }\end{array}$ & $\begin{array}{l}\text { expression of } \\
\text { the reference } \\
\text { currents }\end{array}$ & $\begin{array}{l}\text { expression or } \\
\text { variables for } \\
\text { only harmonics } \\
\text { compensation }\end{array}$ & $\begin{array}{l}\text { variables for only } \\
\text { reactive power } \\
\text { compensation }\end{array}$ & $\begin{array}{l}\text { variables used for } \\
\text { only the current } \\
\text { unbalance correc- } \\
\text { tion }\end{array}$ & $\begin{array}{l}\text { compatibl } \\
\text { with } 1 \text { or } \\
3 \text { phases }\end{array}$ & $\begin{array}{l}\text { of Ada- } \\
\text { lines }(3 \\
\text { phases) }\end{array}$ & $\begin{array}{l}\text { compu- } \\
\text { tational } \\
\text { costs }\end{array}$ \\
\hline neural conv. p-q & (13) & $\tilde{p}+\tilde{q}$ & $q$ & $p_{0}$ & 3 & 2 & + \\
\hline neural mod. p-q & $(30)$ & $\tilde{p}+\tilde{q}_{\alpha}+\tilde{q}_{\beta}+\tilde{q}_{0}$ & $\bar{q}_{\alpha}+\bar{q}_{\beta}+\bar{q}_{0}$ & $q_{\alpha}+q_{\beta}$ & 3 & 4 & ++ \\
\hline neural active cur & $(50)$ & $\tilde{p}$ & \multicolumn{2}{|c|}{ inherently included } & 3 & 1 & - \\
\hline neural synchr & $(60)$ & (59) & \multicolumn{2}{|c|}{ inherently included } & 1 & 1 & - \\
\hline
\end{tabular}


International Journal of Emerging Electric Power Systems, Vol. 12 [2011], Iss. 5, Art. 1

Table 2: Compensating performances of the different harmonics identification schemes

\begin{tabular}{|c|c|c|c|c|c|c|c|c|c|c|c|c|c|}
\hline \multirow{3}{*}{\begin{tabular}{|l|} 
phase a \\
nonlinear load
\end{tabular}} & \multicolumn{6}{|c|}{$|h 5 / h 1(\%)| h 7 / h 1(\%) \mid h 11 / h 1(\%)$} & \multicolumn{4}{|c|}{$|h 13 / h 1(\%)| h 17 / h 1(\%)$} & \multicolumn{3}{|c|}{ THD (\%) rise time } \\
\hline & bal & |unbal & bal & unbal & bal & unbal & bal & unbal & bal & |unbal & bal & |unbal & $(\mathrm{m}$ \\
\hline & 20 & 10 & 13 & 6 & 7 & 2 & 8 & 3 & 3 & 1 & 36 & 14 & - \\
\hline orig. mod. p-q & 0.46 & 0.42 & 0.55 & 0.52 & 0.54 & 0.51 & 0.47 & 0.42 & 0.15 & 0.22 & 0.88 & 0.71 & 200 \\
\hline orig. active cur & 0.46 & 0.43 & 0.55 & 0.51 & 0.54 & 0.52 & 0.47 & 0.42 & 0.15 & 0.22 & 0.84 & 0.75 & 200 \\
\hline neural c & 0.23 & 0.31 & 0.27 & 0.37 & 0.32 & 0.37 & 0.23 & 0.30 & 0.25 & 0.25 & 0.82 & 0.61 & 200 \\
\hline neural $\bmod \mathrm{p}-\mathrm{q}$ & 0.23 & 0.30 & 0.27 & 0.37 & 0.32 & 0.37 & 0.23 & 0.30 & 0.25 & 0.25 & 0.80 & 0.60 & 60 \\
\hline neural active cur & 0.23 & 0.31 & 0.27 & 0.37 & 0.32 & 0.37 & 0.23 & 0.30 & 0.25 & 0.25 & 0.75 & 0.58 & 40 \\
\hline neural synchr & 0.24 & 0.25 & 0.21 & 0.33 & 0.35 & 0.38 & 0.32 & 0.42 & 0.65 & 0.58 & 0.70 & 0.60 & 32 \\
\hline \begin{tabular}{|l|} 
Limits of the IEEE \\
Std 519-1992
\end{tabular} & $<4$ & $<4$ & $<4$ & $<4$ & $<2$ & $<2$ & $\mid<2$ & $<2$ & $<1.5$ & $<1.5$ & $<5$ & $<5$ & - \\
\hline
\end{tabular}

3-phase electrical grid
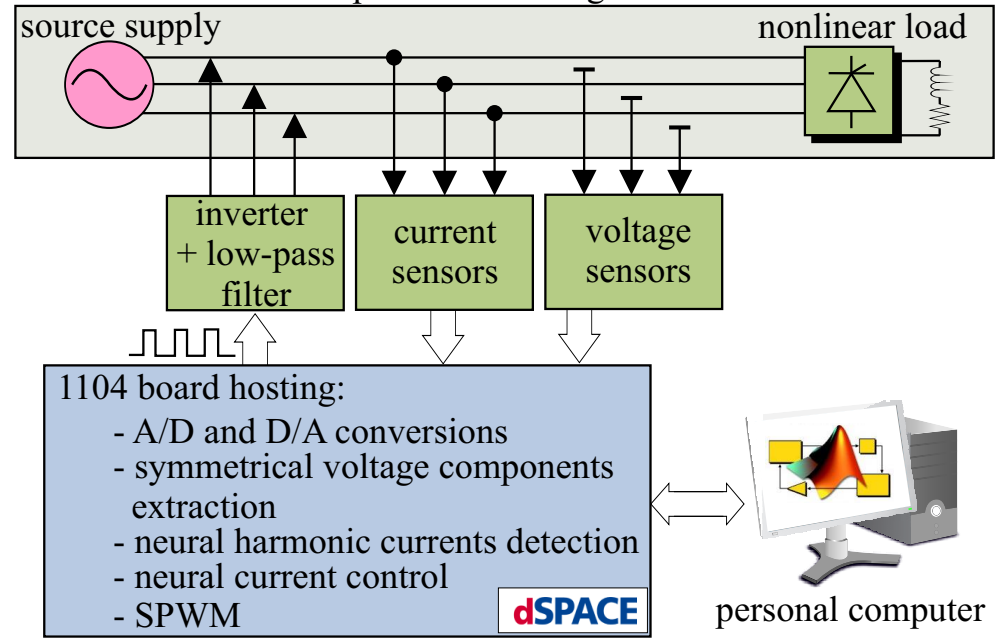

Figure 6: Principle of the experimental test-bed

Table 3: Performance in compensating only for harmonics of rank 5 and 7

\begin{tabular}{|l|c|c|c|c|c|c|}
\hline phase a & \multicolumn{2}{|c|}{$h 5 / h 1(\%)$} & \multicolumn{2}{c|}{$h 7 / h 1(\%)$} & \multicolumn{2}{c|}{$h 13 / h 1(\%)$} \\
\hline nonlinear load & bal & unbal & bal & unbal & bal & unbal \\
\hline load current & 20 & 10 & 13 & 6 & 8 & 3 \\
\hline \hline neural mod. p-q & 0.78 & 0.53 & 0.32 & 0.30 & 6.44 & 3.93 \\
\hline neural active cur & 0.41 & 0.47 & 0.47 & 0.46 & 4.90 & 3.00 \\
\hline neural synchr & 0.63 & 0.54 & 0.38 & 0.35 & 6.24 & 3.85 \\
\hline
\end{tabular}


Nguyen et al.: Harmonics Identification with ANNs : Application to APF

Results are presented by Fig. 7 with a nonlinear load whose parameters change at $t=2.595 \mathrm{~s}$. Fig. 7 a) shows the current waveforms $\mathbf{i}_{L-a b c}$ measured on the nonlinear load. Fig. $7 \mathrm{~b}$ ) shows the currents compensated with a neural scheme based on the conventional p-q powers. The currents compensated with the proposed harmonics identification schemes are represented by Fig. 7 c) to Fig. 7 e). The frequency content of the load current and the compensated currents is represented by Fig. 8. From this frequency spectrum, it is clear that the harmonic currents are efficiently removed. It can also be seen that the performances of the compensating schemes are very close. This is confirmed by the numerical values given by Table 2 . Indeed, this table indicates the amplitude of the harmonic terms of rank 5, 7, 11, 13, and 17 and points out the THD (Total Harmonic Distortion) of each method. Two values are provided, one with the balanced load and one with the unbalanced load after $t=2.595 \mathrm{~s}$. The performances of the neural schemes are compared to those obtained with conventional methods where Adaline are replaced by low-pass filters. In all cases, the neural approaches prove to be more efficient than the conventional methods in terms of reducing the amplitude of the most important harmonic currents but also according to the resulting THD. The harmonic terms are reduced to an amplitude of less than $0.65 \%$ and the THD to less than 0.82 with the neural approaches. Furthermore, Table 2 shows that all schemes permit a full compliance with the IEEE standard 519-1992 which provides maximum harmonics current distortion in percent of the full load fundamental current. The three new neural schemes are faster than the conventional ones and than the neural scheme based on the conventional p-q powers. Indeed, their response time is less than 60 $\mathrm{ms}(60 \mathrm{~ms}$ for the neural method based on the modified $\mathrm{p}-\mathrm{q}$ theory, $40 \mathrm{~ms}$ for the neural active currents method and $32 \mathrm{~ms}$ for the neural synchronized method) where the other methods need $200 \mathrm{~ms}$ to take accound of a frequency content changes.

\subsection{Selective harmonics compensation}

Selective harmonic compensation consists in canceling only some specified harmonic currents. Harmonic compensation by conventional methods is achieved by band-pass filters which introduce additional computational costs, lower precision and time delays. Original methods are therefore not able to precisely compensate for individual harmonic terms. On the other hand, the proposed neural identification schemes allows to precisely estimate each individual harmonic term. 
International Journal of Emerging Electric Power Systems, Vol. 12 [2011], Iss. 5, Art. 1

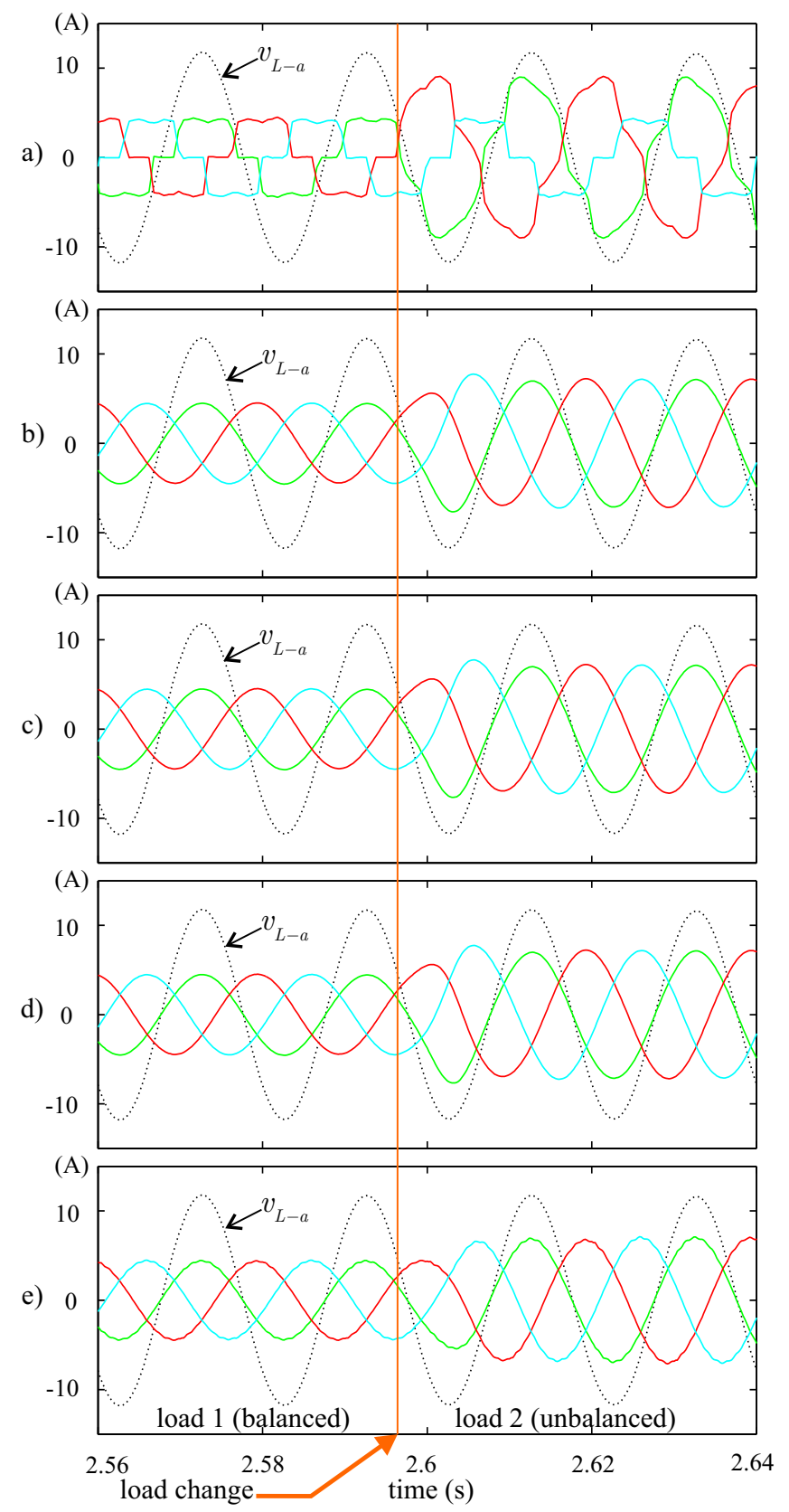

Figure 7: Experimental compensating results with a nonlinear load change at $t=$ 2.595 s., a) three-phase load currents, b) three-phase currents compensated with a neural p-q method, c) three-phase currents compensated with the neural modified p-q method, d) three-phase currents compensated with the neural active currents method, e) three-phase currents compensated with the neural synchronized method 
Nguyen et al.: Harmonics Identification with ANNs : Application to APF
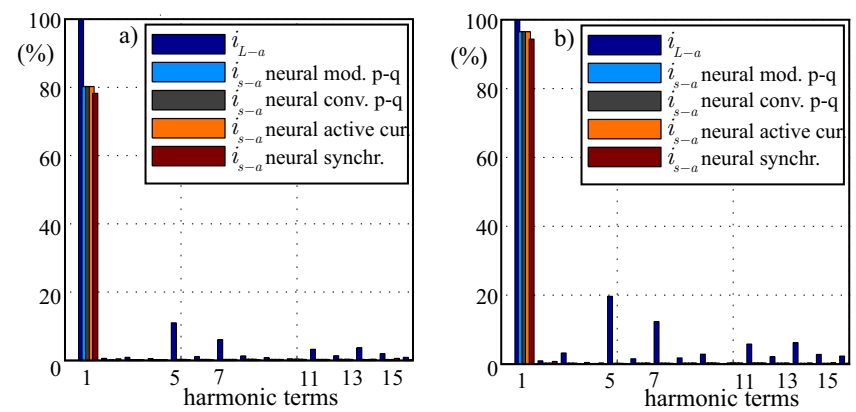

Figure 8: Frequency spectrum of the compensated currents: a) with a balanced nonlinear load, b) under unbalanced conditions after the load variation

As a case study, the objective of the present experiment is to compensate only for the harmonic terms of rank 5 and 7 . The harmonic content generated by the nonlinear load is the same as in the previous experiment. Results presented by Table 3 clearly show that the desired harmonic currents are well compensated under balanced and unbalanced conditions. Indeed, harmonic terms of rank 5 and 7 are reduced to less than $0.8 \%$ whatever the conditions. The harmonic term of rank 13 and other high-order harmonics are not affected. The neural method based on the active curernts is the most efficient in cancelling some specific harmonic terms.

\subsection{Power factor correction}

Power factor correction is achieved by including the terms representative of the reactive power in the compensating currents (see Table 1). The power factors obtained with the APF using the proposed neural schemes are presented in Table 4. It can be seen that the proposed neural schemes permit a full correction of the power factor. The power factor is maintained to a mean value of 0.999 under balanced and unbalanced conditions which is acceptable in industrial installations.

The instantaneous powers $p$ and $q$ consumed by the nonlinear load and the instantaneous powers delivered by the supply and corrected by the APF using the proposed neural schemes are represented by Fig. 9. The instantaneous powers consumed by the nonlinear load fluctuates rapidly. From this figure, it is clear that the correction achieved by proposed neural schemes allows to significantly reduce these fluctuations. The neural methods based on the conventional and modified $p-q$ powers, the neural method based on the active currents and the neural synchronized 
International Journal of Emerging Electric Power Systems, Vol. 12 [2011], Iss. 5, Art. 1

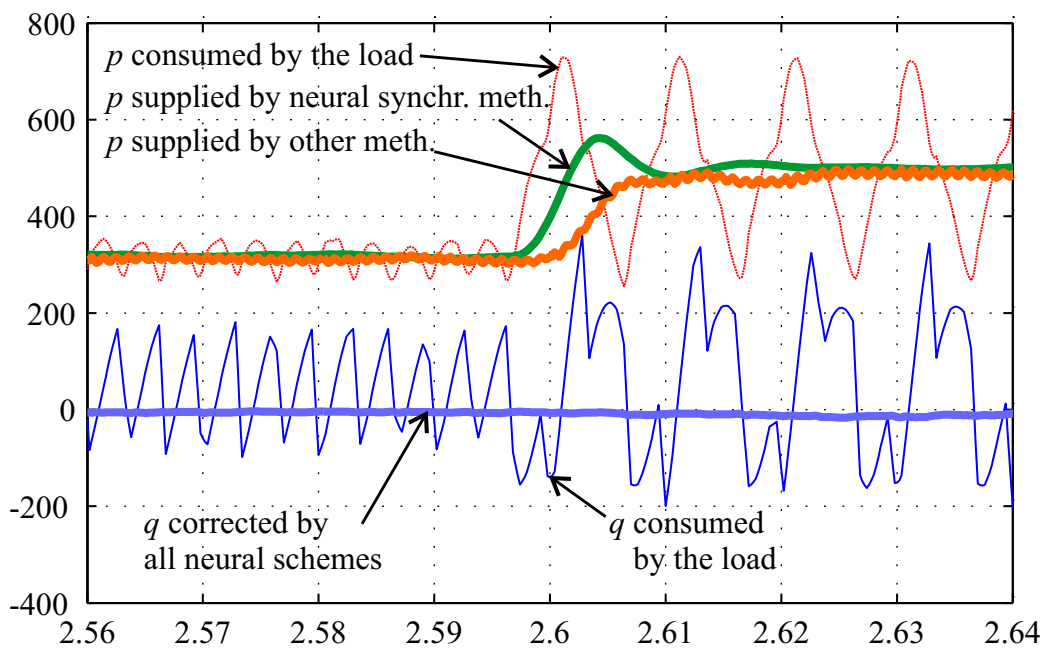

Figure 9: Evolution of the instantaneous powers with and without compensation

Table 4: Comparison of power factor correction

\begin{tabular}{|l|c|c|}
\hline phase a & \multicolumn{2}{|c|}{ power factor } \\
\hline \hline nonlinear load & balanced & unbalanced \\
\hline without correction & 0.760 & 0.750 \\
\hline \hline neural conv. p-q & 0.999 & 0.999 \\
\hline neural mod. p-q & 0.999 & 0.999 \\
\hline neural active cur & 0.999 & 0.999 \\
\hline neural synchr & 0.999 & 0.999 \\
\hline
\end{tabular}

method permit a full compensation of the reactive power, with no difference in the performance appreciable in the time domain analysis. The neural synchronized method leads to lower performance in compensating for the active power compared to the other ones. Except the neural synchronized method, the other neural methods take $50 \mathrm{~ms}$ to fully compensate for the active power.

Finally, the proposed Adaline-based schemes which work in different referenceframes resulting from specific currents or powers decompositions have demonstrated their ability to identify and to track on-line the harmonic currents. The proposed neural schemes lead to performances compliant with the IEEE standard 519-1992 (partially provided by Table 2). Furthermore, a trade-off should be done between best performances, robustness against variations and computational costs. The neural active currents method represents the best solution. It presents the significant advantage of handling the harmonic distortions in a instantaneous power reference frame and optimizing the active currents. Obviously, a high number of harmonics can be taken into account without excessively increasing its computational complexity. 
Nguyen et al.: Harmonics Identification with ANNs : Application to APF

\section{Conclusion}

Three Adaline-based harmonics identification schemes are proposed and designed based on the decomposition of the instantaneous active and reactive powers. One uses the modified p-q theory, one is based on the instantaneous powers and minimizes the reactive currents, and one expresses the harmonic currents in a reference frame synchronized with the direct voltage components. In these methods, Adalines are used to learn and to on-line estimate the harmonic terms of the powers individually. Compensating currents are deduced and used in an active power filtering scheme for on-line canceling the harmonic currents and for correcting the power factor of a power distribution system disturbed by a changing nonlinear load under unbalanced conditions. The performance of the proposed techniques are confirmed by experiments with a dSPACE-based implementation. Results demonstrate the superiority of the neural harmonics identification schemes and that they can be easily inserted in real-time active filtering devices without been computationally extensive.

\section{References}

Akagi, H. (1996): "New trends in active filters for power conditioning," IEEE Transactions on Industry Applications, 32, 1312-1322.

Akagi, H., E. H. Watanabe, and M. Aredes (2007): Instantaneous Power Theory and Applications to Power Conditioning, Wiley-IEEE Press.

Azevedo, H. J., J. M. Ferreira, A. P. Martins, and A. S. Carvalho (2008): "An active power filter with direct current control for power quality conditioning," Electric Power Components and Systems, 36, 587-601.

Czarnecki, L. S. (2004): "On some misinterpretations of the instantaneous reactive power p-q theory," IEEE Transactions on Power Electronics, 19, 828-836.

Dash, P., M. Nayak, M. Senapati, and I. Lee (2007): "Mining for similarities in time series data using wavelet-based feature vectors and neural networks," Engineering Applications of Artificial Intelligence, 20, 185-201.

Dash, P., D. Swain, A. Liew, and S. Rahman (1996): "An adaptive linear combiner for on-line tracking of power system harmonics," IEEE Transactions on Power Systems, 11, 1730-1735.

Flieller, D., D. Ould Abdeslam, P. Wira, and J. Mercklé (2009): "Distortions identification and compensation based on artificial neural networks using symmetrical components of the voltages and the currents," Electric Power Systems Research, $79,1145-1154$. 
Herrera, R. S., P. Salmeron, and H. Kim (2008): "Instantaneous reactive power theory applied to active power filter compensation: Different approaches, assessment, and experimental results," IEEE Transactions on Industrial Electronics, $55,184-196$.

Luenberger, D. G. and Y. Ye (2008): Linear and Nonlinear Programming, International Series in Operations Research and Management Science, volume 116, Springer.

Malengret, M. and C. T. Gaunt (2008): "Decomposition of currents in three- and four-wire systems," IEEE Transactions on Instrumentation and Measurement, $57,963-972$.

Mazumdar, J. and R. G. Harley (2008): "Recurrent neural networks trained with backpropagation through time algorithm to estimate nonlinear load harmonic currents," IEEE Transactions on Industrial Electronics 55, 3484-3491.

Mohanty, M. N., V. Kumar, A. Routray, and P. Kabisatpathy (2010): "Classification of power quality disturbances using parzen kernels," International Journal of Emerging Electric Power Systems 11, article 6.

Moran, L., J. Dixon, and R. Wallace (1995): "A three-phase active power filter operating with fixed switching frequency for reactive power and current harmonic compensation," IEEE Transactions on Industrial Electronics, 42, 402-408.

Nguyen, N. K., D. Ould Abdeslam, P. Wira, D. Flieller, and J. Mercklé (2008): "Artificial neural networks for harmonic currents identification in active power filtering schemes," in 34th Annual Conference of the IEEE Industrial Electronics Society (IECON 2008) Orlando, Florida, USA, 2693-2701.

Nguyen, N. K., P. Wira, D. Ould Abdeslam, and D. Flieller (2009): "Adaptive neural schemes for the control of a shunt active power filter," in 35th Annual Conference of the IEEE Industrial Electronics Society (IECON 2009), Porto, Portugal.

Nguyen, T. and Y. Liao (2009): "Power quality disturbance classification based on adaptive neuro-fuzzy system," International Journal of Emerging Electric Power Systems 10, article 4.

Norgaard, M., O. Ravn, N. Poulsen, and L. Hansen (2000): Neural Networks for Modelling and Control of Dynamic Systems, Advanced Textbooks in Control and Signal Processing, London: Springer-Verlag.

Ould Abdeslam, D., P. Wira, J. Mercklé, D. Flieller, and Y.-A. Chapuis (2007): "A unified artificial neural network architecture for active power filters," IEEE Transactions on Industrial Electronics, 54, 61-76.

Panigrahi, B., P. Dash, and J. Reddy (2009): "Hybrid signal processing and machine intelligence techniques for detection, quantification and classification of power quality disturbances," Engineering Applications of Artificial Intelligence, $22,442-454$. 
Peng, F. Z. (2001): "Harmonic sources and filtering approaches," IEEE Industry Applications Magazine, 7, 18-25.

Picton, P. (2000): Neural Networks, Palgrave Macmillan, 2nd edition.

Widrow, B. and E. Walach (1996): Adaptive Inverse Control, Information and System Sciences Series, Upper Saddle River: Prentice Hall Press.

Willems, J. (1996): "Mathematical foundations of the instantaneous power concepts: A geometrical approach," European Transactions on Electrical Power, 6, 299-304.

Zadeh, M. R. D. (2010): "A real time power system harmonic estimator considering fundamental frequency variations," International Journal of Emerging Electric Power Systems, 11, article 6. 